\title{
Impact of Incentive Therapy on Postural Control in Burn Survivors
}

\author{
Authors \\ Noha F. Mahmoud*, Hoda M. Zakaria**and Wanees M. Badawy** \\ * Department of Physical Therapy for Surgery, Faculty of Physical Therapy, October 6 University. \\ **Department of Physical Therapy for Neuromuscular Disorders and Its Surgery, Faculty of Physical \\ Therapy, Cairo University
}

\begin{abstract}
Background: Burn has a causative factor in avoidant behaviors and lack of postural control.

Objectives: To guide the rehabilitation team making decision regarding the use of incentive therapy via Wii interactive video games in physical therapy practice for burned patients.

Materials and methods: The traditional (control) group received a physical therapy session including positioning, range of motion exercises, stretching exercise for lower limb muscles, and daily walking. The study group (Wii group) received previous physical therapy program followed by 15 minutes of Wii Fit gaming activities.

Main outcome measures: Static and dynamic balance assessment using Biodex Balance system, Timed-Up and Go test, Activities-Specific Balance Confidence Scale and Numeric Pain Rating Scale.

Results: Statistically significant differences were found between the study and control groups; for the favor of patients practiced the Wii interactive video game.

Conclusions: Incentive therapy via active participation of patients using Wii Fit is potentially acceptable as an adjunct to the physical therapy intervention for burned patients aiming to improve postural control.

Keywords: Burn, Incentive therapy, Wii Fit, Biodex Balance system
\end{abstract}

\section{Introduction}

Injury from burns is very common. Hot liquids, flame, electricity and corrosives like acid are the usual causes. In deep burns (where the dermis is burned) new skin cannot grow and the areas heal by scar formation, therefore improperly treated deep burn areas develop burn contractures and become more severe with time ${ }^{[1]}$.
Burn trauma ranges from the minor burn to the devastating injury, It exerts a catastrophic influence on people in terms of human life, including their aesthetic appearance, relationships with others, psychological, social and physical functioning ${ }^{[2,3]}$. Although lower limb burn can cause a significant degree of morbidity. Certainly, foot burns often require prolonged bed rest. Time 
lost from work along with the length of hospitalization and high risk of complications result in a high financial as well as emotional cost to the individual ${ }^{[4]}$.

Patients with lower limb burns suffer not only physically but also mentally, have neuroses which lead to reticence, living a secluded life and formation of an inferiority complex. This influences the formation of personality and future social adaptation by the patient. Following a lower limb burn, gait parameters such as speed, stride length, quality of movement and balance are also affected ${ }^{[3,5]}$. Impaired balance greatly affects the activities of daily living (ADL), independence and gait. Therefore, it's necessary to rapidly attain postural control in order to improve independence, social participation and general health ${ }^{[6]}$.

Burn survivors demonstrate impaired balance in rehabilitation. Balance is a function of multiple factors including tactile sensation, proprioception, muscle strength, joint mobility and cognition. All of these factors are potentially affected in severe burn injury ${ }^{[7]}$. Commercially available interactive video games (IVGs) were not originally designed for rehabilitation purposes but rather for entertainment/gaming and may lack specificity for achieving rehabilitative goals. It has been used for over a decade in a variety of health care settings for rehabilitation of all types of injuries and illnesses. However, most studies are descriptive and objective comparative studies are very limited [8].

Incentive therapy focuses on active participation, self-esteem, motivational features such as score keeping, and competition. The ultimate objective of physiotherapy is for patients to return to full independence and their former occupations ${ }^{[1]}$. Therefore this study was conducted to investigate the impact of incentive therapy using the Nintendo Wii balance board on postural control on burned patients.

\section{Materials and methods}

\section{Subjects:}

This study was conducted in 2014 at the Outpatient clinic of Umm-Almasryeen Hospital, and the assessment was performed at the same place as well as the outpatient clinic of the Faculty of Physical therapy, Cairo University. Thirty male and female subjects (18-40 years) have participated, all of them have healed major lower extremities and lower trunk burns of various surface extent deep second to third degree. They all received the same traditional rehabilitation program during the inpatient period which included positioning, splinting, passive and active stretching exercises, mobility out of bed, gait training, and range of motion exercises (assisted or resisted), all are tailored for each patient individually. The sample was randomly assigned into two groups GI and GII. GI representing the control group consisting of 15 patients at the beginning and ended with 13, received a traditional regular physical therapy program While GII representing the treatment group consisting of 15 patients at the beginning and ended with 14, received beside the traditional regular physical therapy program a specific designed program of interactive video game. Three subjects have been excluded from the study (didn't complete the study to go back to their home outside Giza). Patient selection criteria included the following; patient agreement of participation, lower limb and trunk burn with a total body surface area (TBSA) ranged from $15 \%$ to $45 \%$. Patient exclusion criteria included the following: Not completing the whole duration of the study, not following the physical therapist instructions, any associated lesion of burn, any past history of a neurological or orthopedic condition that may affect balance, any plastic surgery during the period of the study, hearing, visual or cognitive impairments. The randomization schedule was computer-generated using a basic random number generator. All subjects provided informed consent approved by 
the ethical committee of the Faculty of Physical therapy, Cairo University, prior to their participation.

\section{Measurements}

All measurements were done for each patient before and after treatment.

The Biodex Stability System (Biodex corporation, Shirley, NY, USA) was used to assess overall stability index (OASI) and dynamic limit of stability (DLOS). The stability is believed to be the best indicator of the overall ability of the patients to balance performance, in which the larger stability index the greater the degree of instability. It consists of a movable balance platform which provides up to $20^{\circ}$ of surface tilt in a $360^{\circ}$ range and is interfaced with a microprocessor - based actuator. The actuator controls the manually preset degree of surface instability, which ranges from a completely firm surface (stability level 8), to a very unstable surface (stability level 1). The degree to which the platform tilts is dictated by the subject's balance ability. During postural stability testing, the patient's ability to control the platform's angle of tilt is quantified as a variance from center. A large variance is indicative of poor neuromuscular control. During dynamic limits of stability (DLOS) testing, the patient's ability to move from one target to another in a straight line is quantified from a total score of 100 (100 being perfect). For postural stability testing, the degree of surface instability is controlled by microprocessor actuator. The Biodex Balance System allows accurate test and quickly generate a printed report [9].

\section{Patient preparation:}

A detailed sheet was done to every patient. The patient's weight and height were measured and recorded in the evaluation sheet with other information. Patients in the study were given a brief explanation about the procedures using the Biodex Balance System. All patients were asked to avoid anxiety as much as possible, exercises and eating at least two hours before the conduction of the evaluation and treatment protocols, remove the used splints (if any) just before the beginning. To ensure patient safety: each trial was begun with the platform in the locked position. The support rail and biofeedback display were adjusted to ensure patient's comfort and safety. Instructions were given clearly to the nursing staff not to do a restrictive dressing and pressure bandage was used for the lower extremities to prevent potential edema.

Instrument preparation:

The instrument was adjusted as follows: The cable was connected and the instrument was turned on, the Biodex logo screen appeared, all computer functions of the balance system were controlled from the display panel keys. The position of the support handles and its height were adjusted according to the patient's height and comfort. The display height and tilt were adjusted for the patient's comfort. Weight, height and age of the patient were entered to the control screen display located in front of the patient's eyes and the platform firmness was adjusted at level 8 (the most stable level). The foot position and angle for each patient were recorded on the control display. The stance type was adjusted at two leg stance. Assessment of Overall Stability Index:

Patients were encountered to the apparatus and adjust the support handles and visual screen display. Patient was instructed to stand up on the foot platform, grasp the support handles at the beginning of the test. For successive trails at level eight. The patient was asked to try to maintain center position on platform through keeping the cursor on the visual feedback screen. The test trial ended and the platform automatically returned to the locked position. At this point, the balance index reading was displayed reflecting the patient's overall performance in terms of platform deviation from the level position. The data was printed. Additional test trials (second and third) were performed and the mean was calculated.

The data generated from this test was in the form of balance index which include: Overall stability index (OASI) - Anterior/posterior (A/P) index Medial/ lateral (M/L) index. But the overall 
stability index (OASI) was selected in this study. Assessment of Dynamic Limits of Stability: The system was adjusted as before in addition the test difficulty was set at the easiest level (50\% LOS), then the patient was instructed to begin the test and the platform advanced to an unstable surface. The first test trial was then begun. The countdown clock at the lower right of the screen provided a three seconds countdown time. When the countdown completed, the LOS test screen displayed eight boxes arranged around a central box. The boxes on the top portion of the screen represented the anterior medial and lateral limits of stability. The boxes to the left and right of center represented medial- lateral limits of stability. The boxes at the bottom of the screen represent the posterior limits of stability. Once the test begun, the patient tried to move the cursor to the box which appeared on the screen and then back to the center box with as little deviation as possible. The boxes appeared in random order until the patient complete all eight boxes.

Elapsed time was displayed at the bottom of the left side of the screen. Once the patient completed all eight boxes, the clock stopped and the test trial ended, the platform automatically returned to the locked position. The data was printed and additional test trials (second and third) were performed and the mean was calculated.

The data generated were recorded in the form of directional control and time elapsed to complete the test. If all of the patient's movements were directly toward the target straight line, then the amount of extraneous movement would equal zero, and the perfect directional control score was $100 \%$. In other words, high (close to 100\%) directional scores are good, lower scores are worse.

The timed Up and Go (TUG) test was used before and after treatment. TUG test measures, in seconds, the time taken by an individual to stand up from a standard arm chair (approximate seat height of $46 \mathrm{~cm}$ [18 inches], arm height $65 \mathrm{~cm}$. [25.6 inches]), walk a distance of 3 meters (118 inches, approximately 10 feet), turn, walk back to the chair, and sit down. No physical assistance is given. They start with their back against the chair, their arms resting on the armrests, and their walking aid at hand. They are instructed that, on the word "go" they are to get up and walk at a comfortable and safe pace to a line on the floor 3 meters away, turn, return to the chair and sit down again. The subject walks through the test once before being timed in order to become familiar with the test using a stopwatch ${ }^{[10]}$.

Activities-Specific Balance Confidence Scale $(\mathrm{ABC})$ : A questionnaire developed to measure an aspect of the psychological impact of balance impairment and/or falls. The patient was asked to rate his or her confidence in performing each of the activities on a scale from 0 (no confidence) to $100 \%$ (complete confidence) without losing balance or becoming unsteady. The average score obtained is an indicator of balance confidence ${ }^{[11]}$. The Numeric Pain Rating Scale: The patient is asked to make three pain ratings, corresponding to current, best and worst pain experienced over the past 24 hours. The rate is ranged from 0 (no pain) to 10 (worst pain imaginable). The average of the 3 ratings was used to represent the patient's level of pain over the previous 24 hours ${ }^{[12]}$.

\section{Intervention}

Both groups received the traditional physical therapy program in the outpatient clinic which is typically consisted of application of splints, active/passive exercises, stretching of short muscles or tissue and muscles vulnerable to shorting, daily walking and activities of daily living. The exercises and activities were designed and progressed according to patient tolerance and ability. The session extended for almost 40 minutes for all the exercise.

The study group received traditional physical therapy program, in addition to standing balance task on Wii Fit Balance Board: The Wii Fit ${ }^{\mathrm{TM}}$ video game system involves virtual reality programs provide sensory feedback (auditory, visual, and proprioceptive sensations) ${ }^{[13]}$ and has 
built in balance programs that utilize bilateral stance in balance training activities. In this study, the balance board is used to provide visual feedback for balance rehabilitation of burned patients. The Wii system provides feedback to both the therapist and the patient during the course of rehabilitation, objectively displaying the patient's performance. During rehabilitation activities, the Wii system records quantitative information about the subject's balance. Also using for objective measures such as the center of pressure (COP) mean velocity (displacement amplitude over time) and the standard deviation of COP displacement. In order to improve balance, therapists are interested in making their patients shift their weight to different positions. Lateral shifting is generally more important for balance stability. Weight shifting activities are used to give patient practice with maintaining off-center COP postures, thereby increasing their thresholds of stability. With the Wii system, these weight shifting activities involve having patients shift their weight to a target COP range, frequently represented as a light-colored circle. Generally, the patients are required to hold at each position before moving back to a central target location or to a new off-center target ${ }^{[14]}$. The patient started with his weight centered and is asked to shift his COP to a target region that is located forward and to the right. The subject's center of pressure is indicated by the small green circle and the target location is indicated by the larger light blue circle. The subject's trace is displayed to show his path toward the target. Once the subject has maintained his COP within the target region for a sufficient time (usually 5 seconds), the next target is displayed. We chose three programs consisted of Ski Slalom, Table Tilt and Basic step. Ski Slalom, the patient stood on the Wii balance board and shifted the weight right and left to ski through the many flags on the screen, And then, shifting the weight to the front and to the back affecting the speed of the virtual skier. Sharp turns necessitate increased pressure on the side towards which the user wishes the character to turn. Table Tilt, needs very refined weight shifts in all directions in order to move balls across a board and into holes. Basic step, patient step to the front, back, left, and right to help to progress her sense of rhythm. The session of Wii involved video games 15 minutes of balance training, three times/week, for one month. Before asking the patient to maintain static and dynamic postural control, the patient must first take deep breathing while standing still on the balance board and a tracking dot is displayed showing the user's center of gravity (COG) and weight distribution. The user is encouraged to guide the tracking dot into the center of a given target, thereby achieving optimal postural alignment and even weight distribution between the left and right sides.

\section{Statistical analysis}

All statistical tests were performed using SPSS for windows Version 19 (Chicago, IL, USA). All values were expressed as a mean \pm standard deviation (SD) or median (range) value, as appropriate. Non parametric tests (Wilcoxon Signed-ranks, Mann Whitney and Chi-squared tests) were also used. For correlation analysis, Pearson tests were used for variables with normal distribution, and the Spearman test for nonparametric variables. The significant level of $\mathrm{p}<$ 0.05 was considered significant.

\section{Results}

No significant differences in demographical (age and gender) or clinical (TBSA and duration of injury) variables at inclusion were detected between groups (Table 1). No statistically significant differences were found in base line measurements between both groups (Table 2). There were significant differences for all variables before and after treatment for both groups, however the study group (G2) had shown more improvement in proportion to the control group (G1) as shown in table 3 and 4. The correlations between the OSI, ODLOS, TUG, ABC and NRPS in the whole group were shown in table 5. 
Table 1. General characteristics of the patients.

\begin{tabular}{|lccc|}
\hline \multicolumn{1}{|c}{ Variables } & $\begin{array}{c}\text { G1 }(\mathrm{n}=13) \\
\overline{\mathrm{x}}(\mathrm{SD})\end{array}$ & $\begin{array}{c}\text { G2 }(\mathrm{n}=14) \\
\overline{\mathrm{x}}(\mathrm{SD})\end{array}$ & Significance \\
\hline Age (yrs) & $29.6(5.1)$ & $31.3(4.4)$ & $0.361^{\mathrm{a}}$ \\
Gender (M:F) & $4: 9$ & & \\
TBSA (\%) & $25.7(1.5)$ & $26.1(2.1)$ & $0.515^{\mathrm{b}}$ \\
Duration of injury (days) & $26.3(3.5)$ & $29.4(4.9)$ & $0.576^{\mathrm{c}}$ \\
\hline
\end{tabular}

TBSA: Total body surface area; ${ }^{a}$ : significance determined by an independent t-test; ${ }^{\mathrm{b}}$ : significance determined by Chi-squared Test; ${ }^{c}$ : significance determined by Mann-Whitney test.

Table 2. Baseline values of the scores of scales and tests in the assessments carried out before the treatment in both groups.

\begin{tabular}{|lcccc|}
\hline \multicolumn{1}{|c}{ Test } & G1 & G2 & MD & P-value \\
\hline OSI (n) & $\overline{\mathrm{x}}(\mathrm{SD})$ & $\overline{\mathrm{x}}(\mathrm{SD})$ & -0.9 & 0.731 \\
& $8.2(1.3)$ & $9.1(1.3)$ & & \\
ODLOS (\%) & & & -3.5 & 0.079 \\
TUG (s) & $41.7(4.7)$ & $45.2(5.2)$ & -1.6 & 0.200 \\
ABC (\%) & $29.3(2.6)$ & $30.9(3.6)$ & -1.9 & 0.235 \\
$\begin{array}{l}\text { NPRS, median } \\
\text { (range) }\end{array}$ & $51.2(3.9)$ & $53.1(4.2)$ & 0 & 0.61 \\
\hline
\end{tabular}

OSI: Overall stability index; ODLOS: Overall Dynamic Limits of Stability; TUG: Timed Up and Go Test; ABC: Activities-Specific Balance Confidence Scale; NPRS: Numerical Pain Rating Scale, x: Mean; MD: Mean difference; P-value: probability level; *: significant at $\mathrm{p}<0.05$.

Table 3. Comparison between the values of the scores of scales and tests in the assessments carried out before and after the treatment within each group.

\begin{tabular}{|c|c|c|c|c|c|c|c|c|}
\hline \multirow{3}{*}{ Test } & \multicolumn{2}{|c|}{ G1 } & \multirow[t]{3}{*}{ MD } & \multirow[t]{3}{*}{$\mathrm{S}$} & \multicolumn{2}{|c|}{$\mathrm{G} 2$} & \multirow[t]{3}{*}{ MD } & \multirow[t]{3}{*}{ P-value } \\
\hline & $\begin{array}{l}\text { Before } \\
\text { treatment }\end{array}$ & $\begin{array}{l}\text { After } \\
\text { treatment }\end{array}$ & & & $\begin{array}{l}\text { Before } \\
\text { treatment }\end{array}$ & $\begin{array}{l}\text { After } \\
\text { treatment }\end{array}$ & & \\
\hline & $\overline{\mathrm{x}}(\mathrm{SD})$ & $\overline{\mathrm{x}}(\mathrm{SD})$ & & & $\overline{\mathrm{x}}(\mathrm{SD})$ & $\overline{\mathrm{x}}(\mathrm{SD})$ & & \\
\hline OSI (n) & $8.2(1.3)$ & $4.9(1.6)$ & 3.3 & $0.000 *$ & $9.1(1.3)$ & $3.7(1.2)$ & 5.4 & $0.000 *$ \\
\hline ODLOS (\%) & $41.7(4.7)$ & $62.5(3.7)$ & -20.8 & $0.000^{*}$ & $45.2(5.2)$ & $69.6(4.2)$ & -24.4 & $0.000 *$ \\
\hline TUG (s) & $29.3(2.6)$ & $22.4(2.9)$ & 6.9 & $0.000^{*}$ & $30.9(3.6)$ & $19.3(1.3)$ & 11.6 & $0.000 *$ \\
\hline
\end{tabular}




\begin{tabular}{|lllllllll} 
ABC $(\%)$ & $51.2(3.9)$ & $65.4(3.7)$ & -14.2 & $0.000 *$ & $53.1(4.2)$ & $71.3(4.2)$ & -18.2 & $0.000^{*}$ \\
$\begin{array}{l}\text { NPRS, } \\
\text { median } \\
\text { (range) }\end{array}$ & $8(7-9)$ & $6(5-7)$ & 2 & $0.002 *$ & $8(6-8)$ & $5(4-6)$ & 3 & $0.002^{*}$ \\
\hline
\end{tabular}

OSI: Overall stability index; ODLOS: Overall Dynamic Limits of Stability; TUG: Timed Up and Go Test; ABC: Activities-Specific Balance Confidence Scale; NPRS: Numerical Pain Rating Scale, $\bar{x}$ : Mean; MD: Mean difference; P-value: probability level; *:significant at $\mathrm{p}<0.05$.

Table 4. Comparison between the values of the scores of scales and tests in the assessments carried out after the treatment in both groups.

\begin{tabular}{|lcccc|}
\hline \multicolumn{1}{|c}{ Test } & $\begin{array}{c}\text { G1 } \\
\bar{x}(\mathrm{SD})\end{array}$ & $\begin{array}{c}\mathrm{G} 2 \\
\overline{\mathrm{x}}(\mathrm{SD})\end{array}$ & $\mathrm{MD}$ & P-value \\
\hline OSI (n) & $4.9(1.6)$ & $3.7(1.2)$ & 1.2 & $0.036^{*}$ \\
ODLOS (\%) & $62.5(3.7)$ & $69.6(4.2)$ & -7.1 & $0.000^{*}$ \\
TUG (s) & $22.4(2.9)$ & $19.3(1.3)$ & 3.1 & $0.002^{*}$ \\
ABC (\%) & $65.4(3.7)$ & $71.3(4.2)$ & -5.9 & $0.0009^{*}$ \\
NPRS, median & $6(5-7)$ & $5(4-6)$ & 1 & $0.0009^{*}$ \\
(range) & & & & \\
\hline
\end{tabular}

OSI: Overall stability index; ODLOS: Overall Dynamic Limits of Stability; TUG: Timed Up and Go Test; ABC: Activities-Specific Balance Confidence Scale; NPRS: Numerical Pain Rating Scale, x: Mean; MD: Mean difference; P-value: probability level; *:significant at $\mathrm{p}<0.05$.

Table 5. Correlations between the OSI, ODLOS, TUG, ABC and NRPS in the whole group.

\begin{tabular}{|l|l|l|l|l|l|l|}
\hline \multicolumn{2}{|l}{ Correlations } & \multicolumn{2}{c}{ OSI } & ODLOS & TUG & ABC \\
\hline OSI & $\mathrm{r}$ (p-value $)$ & & $-0.62(0.02)^{*}$ & $0.59(0.03)$ & $-0.57(0.04)^{*}$ & $-0.56(0.05)^{*}$ \\
\hline ODLOS & $\mathrm{r}(\mathrm{p}$-value $)$ & $-0.62(0.02)^{*}$ & & $0.577(0.04)^{*}$ & $0.56(0.05)^{*}$ & $-0.60(0.03)^{*}$ \\
\hline TUG & $\mathrm{r}(\mathrm{p}$-value $)$ & $0.59(0.03)^{*}$ & $0.577(0.04)^{*}$ & & $0.72(0.006)^{*}$ & $-0.69(0.02)^{*}$ \\
\hline ABC & $\mathrm{r}(\mathrm{p}$-value $)$ & $-0.57(0.04)^{*}$ & $0.56(0.05)^{*}$ & $0.72(0.006)^{*}$ & & $-0.67(0.02)^{*}$ \\
\hline NRPS & $\mathrm{r}(\mathrm{p}$-value $)$ & $-0.56(0.05)^{*}$ & $-0.60(0.03)^{*}$ & $-0.69(0.02)^{*}$ & $-0.67(0.02)^{*}$ & \\
\hline
\end{tabular}


OSI: Overall stability index; ODLOS: Overall Dynamic Limits of Stability; TUG: Timed Up and Go Test; ABC: Activities-Specific Balance Confidence Scale; NPRS: Numerical Pain Rating Scale, *:significant at $\mathrm{p}<0.05$.

\section{Discussion}

Physical therapy is a critical, though painful, component of burn rehabilitation therapy. Early and aggressive physical therapy can help counter the decreased range of motion and severe contractures that can develop secondary to burn injury or associated skin grafting ${ }^{[15]}$. As a result, burn rehabilitation therapy is often key to improve functional outcome and minimize long-term disability. Unfortunately, patients may be reluctant to participate fully in physical therapy due to the significant procedural pain that can be triggered by any activity that are intended to help them heal $[16,17]$

Initial and forceful physical therapy can help opposethe contractures and limited joint mobility that can result from hypertrophic scarring and decreased skin elasticity as the body tries to recover after burn injury. As a result, burn rehabilitation therapy is often introduced to maximize functional outcome and minimize longterm disability. Unsuccessfully, burned patients may be unwilling to participate fully in physical therapy program due to the noticed procedural pain that can be evoked by simple activities that are proposed to help them make well ${ }^{[15]}$.

The Wii group showed improvement in pain perception seen as decreased NRPS values.in agreement with ${ }^{[18,19,20]}$. Subjective improvement in mood, coupled with a decrease in pain perception during burn rehabilitation, may have important implications for patient obedience with burn rehabilitation activities. Since anticipatory anxiety can be coupled with repeated painful procedures and may add to a patient's subjective experience of pain $^{[21]}$. And this can be seen in the moderate correlation between the pain scale scores in one side and the balance and functional test measurements. The active participation of the patients via incentive therapy creates a nonpharmacologic form of analgesia by changing the activity of the body's intricate pain modulation system which in turn enables individuals to immerse themselves in a virtual world ${ }^{[19]}$. On the other hand, a study by Fung et al., ${ }^{[2]}$ found no significant differences in changes in pain following a rehabilitation program using Nintendo Wii Fit.
However, their study performed this program on post-operative total knee replacement patients.

The efficacy of virtual reality has not been proven and the exact mechanisms behind virtual reality's action remain unknown ${ }^{[19]}$.Optimal postural balance (PB) is the main foundation for the individual's ability to perform movement, and composes a central element in ensuring adequate movement capabilities. It is no longer believed simply as summation of static reflexes but rather is believed a complex skill based on the interaction of dynamic sensorimotor processes. Poor postural control increases the risk of falls. Falls are a major problem and cause not only various physical injuries but are also associated with high medicalrelated costs. So, accurate and reliable measures of PB in scientific and clinical settings are necessary to prevention of problems caused by falls ${ }^{[23]}$. In recent times, immersive virtual reality has been recommended as a supplementary choice. This treatment may be to a great degree effective because it offers an interactive, computergenerated virtual setting that is highly distracting to the patient ${ }^{[24]}$.

Incentive therapy increases cognitive function and therefore optimizes the ability to understand the things that occur in daily life such as attention, memory and executive function. Cognitive-motor interference refers to the phenomenon that occurs when one or two tasks that interfere with each other are being performed, such as engaging in cognitive and motor tasks simultaneously. Motor performance and learning can be facilitated under some dual-task circumstances for young healthy adults by the use of attention resources that improved the encoding of the primary motor task. Adding a secondary cognitive task creates better balance performance than single task ${ }^{[13]}$.

The noticeable improvement of balance in burn survivor patient who trained by incentive therapy via Nintendo Wii in static and dynamic balance measured by the Biodex balance system may be attributed to the Wii Fit games as it encourage lower extremity movement, challenge balance, and require the patient to remain in a standing position during play. These activities have the potential to address rehabilitation goals involving recovery of 
lower extremity function and maintenance of symmetrical weight bearing on both legs. This is agreed with McGough et al., 2011 ${ }^{[25]}$ who previously reported that the asymmetries of the weight shift got better by using Nintendo Wii.

Another study supported that reasoning of improved balance; is conducted by Deutsch et al., 2008, who studied the use of a low-cost, commercially available gaming console (Wii) for rehabilitation of an adolescent with cerebral palsy as in the present study. They stated that the weight distributions of the lower limbs come into more symmetrical, and the child had a decrease in postural sway, indicating that there was an increase in stance stability ${ }^{[26]}$. In addition; there was a positive effect of the device in increasing the level of confidence $(\mathrm{p}=0.0009)$ and a strong correlation with the timed up and go test (as a functional activity) ( $\mathrm{r}=0.72, \mathrm{p}=0.006)$, as the Wii facilitate certain cognitive skills like controlling functions, visual and attentional skills and it could be an effective tool in cognitive training.

Complications of severe burn injuries including prolonged hospitalization, poor nutrition, pain and neuropathies can further worsen balance. Impaired balance retards functional mobility, safety and ultimately independence. Incentive therapy can offer ways to simulate sport, recreational and functional activities without the intensity or risk associated with performing the actual activity.

\section{Conclusion}

This preliminary randomized controlled trial suggests that incentive therapy using Wii Fit is recommended for use as an adjunct to physical therapy treatment in the rehabilitation of balance and lower extremity function in burned survivors. It is a therapeutic intervention option that is considered to be more fun and interesting than conventional exercise. Additional studies with larger samples with different degrees of burn may help to verify the degree to which the use of Wii Fit as a physical therapy adjunct plays a role in other aspects of rehabilitation rather than balance.

\section{References}

1. Okhovatian F, Zoubine N. A comparison between two burn rehabilitation protocols. Burns. 2007: 33: 429-434.

2. Falder S, Browne A, Edgar D, Staples E., Fong J, Rea S, Wood F. Core outcomes for adult burn survivors: A clinical overview. Burns. 2009: 35: 618- 641.

3. Shakirov B. Deep foot burns: Effects of early excision and grafting, Burns. 2011: 37: 1435- 1438.

4. Hemington-Gorse S, Pellard S, WilsonJones N, Potokar T. Foot burns: Epidemiology and management. Burns. 2007: 33: 1041-1045.

5. Grisbrook TL, Reid SL, Elliott CM, Elliott BC, Edgar DW, Wood FM. Lower limb functional outcome assessment following burn injury: A novel use for 3D laboratorybased movement analysis. Burns. 2010: 36: e24- e30.

6. Januario F, Campos I, Amaral C. Rehabilitation of postural stability in ataxic/hemiplegic patients after stroke. Disability and Rehabilitation. 2010: 32 (21):1775-9.

7. Schneider JC, Qu HD, Lowry J, Walker J, Vitale E, Zona M. Efficacy of inpatient burn rehabilitation: A prospective pilot study examining range of motion, hand function and balance. Burns. 2012: 38:164171.

8. Parry I.S, Bagley A, Kawada J., Sen S, Greenhalgh D.G., Palmieri T.L.: Commercially available interactive video games in burn rehabilitation: therapeutic potential. Burns.2012: 38: 493 - 500.

9. Testerman, C. and Griend, R.: Evaluation of ankle instability using the Biodex stability system. Foot and Ankle Intentional. 1999: 20(5): 317-321.

10. Cattaneo D, Jonsdottir J, Repetti S. Reliability of four scales on balance disorders in persons with multiple sclerosis. DisabilRehabil. 2007: Dec 30; 29(24):1920-5.

11. Miller WC, Deathe AB, Speechley M. Psychometric properties of the Activitiesspecific Balance Confidence Scale among individuals with a lower-limb amputation. 
Arch Phys Med Rehabil. 2003: May 84(5): 656-61.

12. Williamson A. Pain: a review of three commonly used pain rating scales. Issues in Clinical Nursing. 2005: 14: 798-804.

13. Bayouk JF, Boucher JP, Leroux A. Balance training following stroke: effects of taskoriented exercises with and without altered sensory input. Int J Rehabil Res. 2006: 29: 51-9.

14. Gil-Gómez JA, Lloréns R, Alcañiz M, Colomer C. Effectiveness of a Wii balance board-based system (eBaViR) for balance rehabilitation: a pilot randomized clinical trial in patients with acquired brain injury. $\mathbf{J}$ NeuroengRehabil. 2011: May 23: 8:30. doi: 10.1186/1743-0003-8-30.

15. Schmitt YS, Hoffman HG, Blough DK, Patterson DR, Jensen MP, Soltani M, Carrougher GJ, Nakamura D, Sharar SR. A Randomized, controlled trial of immersive virtual reality analgesia during physical therapy for pediatric burn injuries. Burns. 2011: Feb 37(1): 61-68.

16. Ehde DM, Patterson DR, Fordyce WE. The quota system in burn rehabilitation. J Burn Care Rehabil. 1998;14:436-440.

17. Patterson DR, Sharar SR. Burn pain. In: Loeser JD, Butler SH, Chapman CR, Turk DC, editors. Bonica's management of pain. Philadelphia, PA: Lippincott; 2001.

18. Hoffman HG, Patterson DR, Seibel E, Soltani M, Jewett-Leahy L, Sharar SR. Virtual reality pain control during burn wound debridement in the hydrotank.Clin J Pain. 2008: May;24(4):299-304.

19. Mahrer NE, Gold JI. The use of virtual reality for pain control: a review. Curr. Pain Headache Rep. 2009: Apr;13(2):100-9.

20. CarrougherGJ, Hoffman HG, Nakamura D, Lezotte D, Soltani M, Leahy L, EngravLH, Patterson DR. The effect of virtual reality on pain and range of motion in adults with burn injuries.J Burn Care Res. 2009: $\quad$ Sep-Oct;30(5):785-91.doi: 10.1097/BCR.0b013e3181b485d3.

21. Palermo TM, Drotar D. Prediction of children's postoperative pain: the role of presurgical expectations and anticipatory emotions. J Pediatr Psychol. 1996: 21:683698.
22. Fung V, Ho A, Shaffer J, Chung E, and Gomez M. Use of Nintendo Wii Fit ${ }^{\mathrm{TM}}$ in the rehabilitation of outpatients following total knee replacement: a preliminary randomised controlled trial. Physiotherapy. 2012: 98: 183-188.

23. Kwon Y, Choi Y, Nam S, Lee M. The Influence of Time of Day on Static and Dynamic Postural Control in Normal Adults. J Phys. Ther Sci. 2014: Mar 26(3): 409-412.

24. Hoffman HG, Doctor JN, Patterson DR, Carrougher GJ, Furness TA., 3rd Virtual reality as an adjunctive pain control during burn wound care in adolescent patients. Pain. 2000: 85(1-2):305-309.

25. McGough R, Paterson K, Bradshaw E, Bryant A, Ross C. The Use Of Nintendo Wii Balance Board For Real-Time Investigation Of Weight Bearing Asymmetry. Portuguese Journal of Sport Sciences. 2011:11 (Suppl.2).

26. Deutsch JE, Borbely M, Filler J, et al. Use of a low-cost, commercially available gaming console (Wii) for rehabilitation of an adolescent with cerebral palsy. PhysTher. 2008: 88: 1196-07. 